\title{
Radiation reaction effects in cascade scattering of intense, tightly focused laser pulses by relativistic electrons: Classical approach
}

\author{
A. Zhidkov, ${ }^{1,2, *}$ S. Masuda, ${ }^{1,2}$ S. S. Bulanov, ${ }^{3}$ J. Koga, ${ }^{4}$ T. Hosokai, ${ }^{1,2}$ and R. Kodama ${ }^{1,5}$ \\ ${ }^{1}$ Photon Pioneers Center, Osaka University, 2-1, Yamadaoka, Suita, Osaka 565-0871, Japan \\ ${ }^{2}$ Japan Science and Technology Agency, CREST, 2-1, Yamadaoka, Suita, Osaka 565-0871, Japan \\ ${ }^{3}$ University of California, Berkeley, California 94720, USA \\ ${ }^{4}$ Quantum Beam Science Directorate, JAEA, Kizugawa, Kyoto 619-0215, Japan \\ ${ }^{5}$ Graduate School of Engineering; Institute of Laser Engineering, Osaka University, \\ 2-1, Yamadaoka, Suita, Osaka 565-0871, Japan
}

(Received 6 August 2013; published 13 May 2014)

\begin{abstract}
Nonlinear cascade scattering of intense, tightly focused laser pulses by relativistic electrons is studied numerically in the classical approximation including radiation damping for the quantum parameter $\left\langle\hbar \omega_{\mathrm{x} \text { ray }}\right\rangle / \varepsilon<1$ and an arbitrary radiation parameter $\chi$. The electron's energy loss, along with its being scattered to the side by the ponderomotive force, makes scattering in the vicinity of a high laser field nearly impossible at high electron energies. The use of a second, copropagating laser pulse as a booster is shown to partially solve this problem.
\end{abstract}

DOI: 10.1103/PhysRevSTAB.17.054001

PACS numbers: 41.60.-m, 41.75.Jv

\section{INTRODUCTION}

The scattering of intense laser light by relativistic electrons, especially in cascade regimes where an electron scatters more than one x-ray photon, is one of the most important mechanisms of radiation production from plasma produced by intense femtosecond laser pulses [1]. Presently, laser intensities are approaching a threshold where the radiation becomes dominant [2]. Therefore, this radiation dominant regime has become an object of detailed study both experimentally and theoretically. So far, ab initio simulations of the radiation even from a classical relativistic plasma standpoint are impossible due to the requirements of extremely high spatial and temporal resolution. Understanding radiation processes in relativistic systems in high laser fields is a pressing issue for the derivation of proper empirical approaches to calculate radiation damping and spectra for such plasma. Nonlinear and quantum radiation effects in the interaction of relativistic electron beams and intense laser pulses occur at relatively smaller, accessible laser intensities. Their experimental and numerical study could result in the development of the necessary radiation models.

In contrast to cyclotron radiation [3], the laser light scattering process is always a free-free electron transition and may proceed differently depending on the field and electron energies. At low laser pulse intensities and high electron energies there is the well-known Compton

"zhidkov@ppc.osaka-u.ac.jp

Published by the American Physical Society under the terms of the Creative Commons Attribution 3.0 License. Further distribution of this work must maintain attribution to the author $(s)$ and the published article's title, journal citation, and DOI. scattering that is determined only by the quantum parameter: $\nu=2 \gamma_{0} \hbar \omega_{0} / m c^{2}$, where $\gamma_{0}$ is the electron relativistic factor taken to be $\gamma_{0} \gg 1$ and $\omega_{0}$ is the frequency of a laser photon (see details in Ref. [4]). If the parameter $\nu$ exceeds unity, the maximum energy of the x-ray photon must be less than $4 \gamma_{0}^{2} \omega_{0}$, and the photon is emitted at an angle smaller than $\pi$ in the propagation direction of the electron. The scattering cross section decreases with further increase in $\gamma_{0}$ [4]. At high intensities the "quantum" scattering is usually characterized by the parameter: $\chi=\left(\hbar \omega_{0} \gamma / m c^{2}\right) \sqrt{(\tilde{\vec{E}}+\vec{v} \times \tilde{\vec{B}} / c)^{2}-(\vec{v} \cdot \tilde{\vec{E}} / c)^{2}}[5,6]$, where $\vec{E}$ and $\vec{B}$ are normalized: $\tilde{\vec{E}}=e \vec{E} / m c \omega=\vec{a}_{0}$. For $a_{0} \gg 1: \chi \sim \hbar \omega_{0} \gamma a_{0} / m c^{2}$. For $\chi \gg 1$ the scattering probability, $P$, differs from the classical one as $\sim P_{\text {classical }}(\chi) \chi^{-1 / 3}$ [5]. However for $a_{0} \ll 1$, this parameter differs from the quantum parameter, $\nu$, used for Compton scattering [4]. Moreover, the parameter $\chi^{2}$ fits amazingly well with the largest part of the classical radiation force responsible for scattering when $\gamma_{0} \gg 1$ [7]. If the parameter $\chi$ is large, the approximation of a "fixed" electron trajectory as in Ref. [8] is no longer valid; radiation damping becomes essential. Depending on the pulse duration $\tau$, it may be a single event resulting in the electron retardation or a cascade of scattering events with the same result. The simplest estimation of the number of scattered photons $n_{\mathrm{ph}}=\pi\left(m c^{2} / e\right)^{2}\left(I \tau / \hbar \omega_{0}\right)$, where $I$ is the laser pulse intensity, gives $n_{\mathrm{ph}} \sim 2-3$, or the cascade, already for an $E \sim 1 \mathrm{~J}$ energy laser pulse focused in a $w_{0} \sim 10 \mu \mathrm{m}$ focus spot.

In a strong laser field, when $a_{0} \geq 1$, harmonics appear and the quantum parameter is different from the standard one. Its definition is not simple. In the fixed trajectory, 
classical approximation the spectrum is flat till the energy is less than the critical energy [8]:

$$
\hbar \omega_{c}=\frac{3 a_{0}^{3} \hbar \omega_{0}}{4 \sqrt{2}}\left[\frac{\gamma_{0}^{2}(1+v / c)^{2}}{1+a_{0}^{2} / 2}+1\right]
$$

where $v$ is the electron velocity. At high frequencies the spectrum exponentially decreases, $S(\omega) \sim \exp \left(-2 \omega / \omega_{c}\right)$. The simplest definition of the quantum condition might be as follows: $\hbar \omega_{c} \gg m c^{2}\left(\gamma_{0}-1\right)$, or for large $a_{0}$ and $\gamma_{0}$ as $a_{0} \gg\left(\sqrt{2} m c^{2} / 6 \hbar \omega_{0}\right) / \gamma_{0}$ (for an x-ray free electron laser this condition is achieved for approximately $10^{4}$ times lower $a_{0}$ ), when most of the radiation energy should be emitted with an essential recoil: parametrically for large $a_{0}$ this condition coincides with $\chi \gg 1$. However, radiation damping may change the spectra and result in softer quantum conditions for the total radiation power.

The interaction of an electron with an intense laser pulse is not always a stationary process, which can be characterized by a cross section or a probability. For example, a typical quantum consideration of the problem $[5,6,9,10]$ assumes that the electron instantly appears in a strong plane wave propagating contrary to the wave with momentum $p_{0}$. This approach is built on a relatively simple basis of Volkov's functions, $\psi_{p}^{V}=A e^{i S / \hbar}$, where $A$ is an amplitude and $S$ is the classical action [4,5]. The calculated probability naturally includes radiation reaction. However, in a strong field an electron will lose its energy rapidly and, then, the electron will be trapped and move along the wave. Therefore, the equations for the scattering matrix

$\partial C_{p} / \partial t=-\int d p^{\prime} \psi_{p}^{V} e \hat{\alpha} \hat{A}_{\operatorname{Rad}} \bar{\psi}_{p^{\prime}}^{V} C_{p^{\prime}}, \quad C_{p}(t=-\infty)=\delta_{p p_{0}}$,

where $A_{\text {Rad }}$ is the vacuum radiation potential [4], have no small parameter; their solution, $C_{p}(t \gg \tau)$, restricted by the pulse duration, may have a quite broad distribution over momentum, $p$, making the exact solution not achievable in the cascade regime. Moreover, a relativistic electron is a classical particle with a certain trajectory undergoing ponderomotive scattering, which is missing in the quantum approach. The classical consideration including radiation damping, therefore, may help in determining the physical model necessary for an advanced quantum consideration.

In this paper, the scattering of intense focused laser light and its effects on the electron motion is analyzed in the framework of the classical approximation [11] including radiation damping and ponderomotive effects.

\section{RADIATION DAMPING FORCE AND SPECTRA}

A sixth-order Runge-Kutta method [12] is used to solve the equations of motion for an electron written in the form

$$
\begin{aligned}
\frac{d \vec{p}}{d t} & =-e[\vec{E}+\vec{p} \times \vec{B} /(m c \gamma)]-\vec{f}_{\mathrm{RD}} ; \\
\gamma & =\sqrt{1+\vec{p}^{2} /(m c)^{2}}, \\
\frac{d \vec{r}}{d t} & =\vec{p} /(m \gamma),
\end{aligned}
$$

where $\vec{p}, \vec{r}$ are the electron momentum and coordinate, $f_{\mathrm{RD}}$ is the radiation force in the form of Ref [4] with the main part responsible for the light scattering given as

$$
\vec{f}_{\text {scatt }}=-\frac{2 e^{4}}{3 m^{2} c^{5}} \gamma^{2} v\left\{\left(\overrightarrow{\boldsymbol{E}}+\frac{1}{c} \overrightarrow{\boldsymbol{v}} \times \overrightarrow{\boldsymbol{H}}\right)^{2}-\frac{1}{c^{2}}(\overrightarrow{\boldsymbol{v}} \cdot \overrightarrow{\boldsymbol{E}})^{2}\right\},
$$

with $\vec{E}$ and $\vec{B}$ the electric and magnetic fields, respectively, and $\vec{v}$ is the electron velocity. To correctly include the ponderomotive force, we use the parabolic approximation for the focused laser field [13]. The transverse components have the following forms:

$$
\begin{aligned}
E_{\perp}(r, t)= & \exp \left[-(z / c-t)^{2} / \tau^{2}\right] \sum\left[\operatorname{Re} E_{l, m}^{0} H_{l}\left(\frac{\sqrt{2} x}{w(z)}\right)\right. \\
& \times H_{m}\left(\frac{\sqrt{2} y}{w(z)}\right) \frac{w_{0}}{w(z)} \times \exp \left\{-\left(x^{2}+y^{2}\right) / w(z)^{2}\right. \\
& +i[k z-\omega t-(l+m+1) \phi(z) \\
& \left.\left.\left.+k\left(x^{2}+y^{2}\right) / 2 R(z)\right]\right\}\right]
\end{aligned}
$$

where the transverse component of the magnetic field equals $\vec{B}_{\perp}= \pm \vec{E}_{\perp}$ depending on the propagation direction, $w(z)=w_{0} \sqrt{1+\frac{z^{2}}{L_{R}^{2}}}, R(z)=z\left(1+\frac{L_{R}^{2}}{z^{2}}\right), \varphi(z)=\tan ^{-1}\left(z / L_{R}\right)$, and $L_{R}=\pi w_{0}^{2} / \lambda$ is the Rayleigh length, $w_{0}=\pi f \lambda / 2 D(f$, $D$, and $\lambda$ are the focal length, beam diameter, and the pulse wavelength), and $H_{l}$ is a Hermite polynomial. The longitudinal components satisfy the equations $\operatorname{div} \vec{E}=0$ and $\operatorname{div} \vec{B}=0$. In particular, for the pair $\left\{E_{\mathrm{x}}, B_{\mathrm{y}}\right\}$ : $E_{\mathrm{z}}-x\left[Q(z) / L_{\mathrm{R}}\right] E_{\mathrm{x}}, B_{\mathrm{z}}=-y\left[Q(z) / L_{\mathrm{R}}\right] E_{\mathrm{x}}$, and $Q(z)=$ $-\mathrm{i} \frac{w_{0}^{2}}{w(z)^{2}}+\frac{L_{\mathrm{R}}}{R(z)}$. Calculations are performed for $l=m=0$ and $w_{0}=5 \mu \mathrm{m}$. To calculate the spectrum (for the intensity), we exploit the well-known equation for the Lienard-Wiechert potential $[7,8]$

$$
\begin{aligned}
\frac{d^{2} S\left(\omega_{\mathrm{x} \mathrm{ray}}, \vec{\Omega}\right)}{d \omega_{\mathrm{x} \mathrm{ray}} d \vec{\Omega}}= & \frac{e^{2}}{\pi^{2} m^{2} c^{3}} \mid \sum\left[\vec{n} \times\left(\vec{n} \times \vec{v}_{j}\right)\right] e^{i \omega_{\mathrm{x} \mathrm{ray}}\left[t-\vec{n} \vec{r}_{j} / c\right]} \\
& \times\left.\frac{\sin \left(0.5 \omega_{\mathrm{x} \mathrm{ray}} \Delta t\left(1-\vec{n} \vec{v}_{j} / c\right)\right.}{\left(1-\vec{n} \vec{v}_{j} / c\right)}\right|^{2},
\end{aligned}
$$

where $r_{\mathrm{j}}, v_{\mathrm{j}}$ are the electron coordinate and velocity, respectively, at $t=t_{\mathrm{j}^{\prime}}$, and $\Delta t$ is the integration time step 
$\left(\sim 10^{-5} \omega_{0}^{-1}\right)$. The spectral resolution is given by the number of spectral points, $M$, where $M=2000$ with the maximum frequency $\omega_{\text {x ray }}{ }^{\max }=(5 / 10) a_{0} \omega_{0}\left(4 \gamma_{0}^{2}\right)$. This choice cannot provide enough resolution at lower frequencies. However, it is good enough to describe the spectra of the highest harmonics, which are necessary to estimate the contribution of quantum effects to the total amount of radiation losses.

\section{SIMULATION RESULTS}

In Fig. 1 the typical spectra of backward scattering with respect to the laser propagation direction $(\theta=\pi)$ are presented for $a_{0}=1$ for Gaussian pulses of duration 20, 90, and 200 fs with $a_{0}=1$ for a head-on collision with $\gamma_{0}=200$. These spectra, as expected, demonstrate the proportional increase of intensity of the scattered $x$ rays with the pulse duration. However, even in this weakly nonlinear case, the spectra depend on the pulse duration. For the long laser pulse the spectrum is very similar to that which follows from the fixed trajectory approximation as in Ref. [8]. For shorter pulses, a certainly stronger red shift is clearly seen in the spectrum. This means that the cascade regime is not simply a sum of independent events of Compton scattering. The study of this effect is out of the scope of this paper. We also refer the readers to Refs. [11,14] where this dynamic mass shift effect is discussed.

The backward spectra for the high intensity laser pulses $a_{0}=200$ are presented in Figs. 2(a) and 2(c) also for

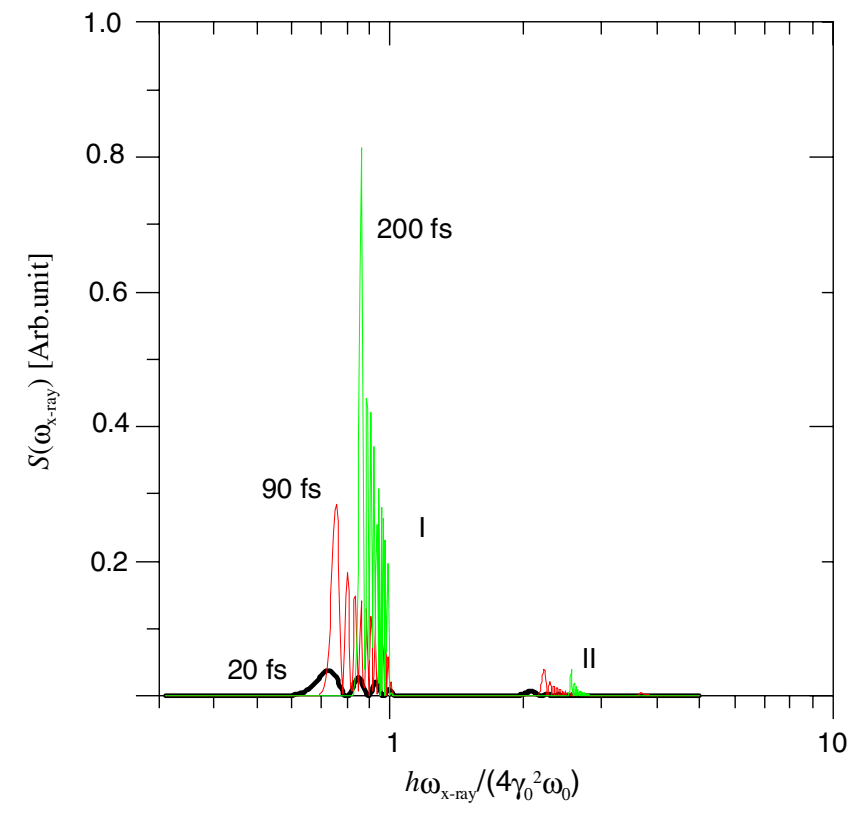

FIG. 1. Spectra of backward scattering $(\theta=\pi)$ at $a_{0}=1$ for Gaussian pulses of duration 20, 90, and $200 \mathrm{fs}$; head-on collision at $\gamma=200$. (I) are the fundamental harmonics, and (II) the third harmonics (even harmonics for the linear polarized laser pulse vanish). Radiation damping is included. head-on collisions with and without radiation damping. There is a clear dependency on the pulse duration; the number of scattered photons not only increases, but there also is a clear difference in the spectral distribution. The radiation damping $(\mathrm{RD})$ drastically changes the resulting spectra. The intensity decreases by an order of magnitude when RD is included. Since the radiation of a single electron is considered for a nonuniform laser field with a limited spectral resolution, the spectrum shape is not smooth. However this is not noise; calculations with very different temporal resolution $\left(\omega_{0} \Delta t\right.$ ranging from $10^{-7}$ to $10^{-4}$ ) give exactly the same results. The corresponding evolution of electron momenta for these cases are shown in Figs. 2(b) and 2(d). One can see that the radiation in the vicinity of the highest field occurs at electron energies much lower than its initial.

Grey squares exhibit the spectral area where the recoil effect is very strong. Even without RD the integral energy in the quantum range shown in the figure is essentially less than that from the lower frequencies emitted indicating no serious recoil effect. With RD the scattered photons lie far from the quantum area making the quantum contribution softer and, therefore, making the classical approach more accurate. In Fig. 3 the parameter $\chi$ is calculated for a relatively high electron energy $\varepsilon \sim 1 \mathrm{GeV}$ and strong field with and without RD. One can see that the parameter $\chi$ reaches $\sim 1$ in the absence of $\mathrm{RD}$ and a considerable part of the spectra could require a quantum approach. However according to $\operatorname{Ref}[5]$, the ratio $\mathrm{P} / \mathrm{P}_{\text {classical }} \approx \chi^{-1 / 3}$ decreases slowly with $\chi$, and, therefore, the total radiated power should essentially not differ from the classical one even in this case. Moreover, RD results in a much lower $\chi$ (the maximum value of $\chi \sim 0.35$ is reached for a very short time, while $\langle\chi\rangle \sim 0.2$ ) by almost an order of magnitude making the classical calculation for the total radiated power quite correct. The spectrum for $\varepsilon \sim 1 \mathrm{GeV}$ and different pulse durations are given in Fig. 4 for $a_{0}=100$ including RD. The difference in the spectrum cannot be expressed by simple probability approaches; while the nonlinear part does not change much, the linear part of the spectrum changes dramatically. The increase of $a_{0}$ up to 500 amazingly results in a smaller number of scattering photons because the main interaction zone occurs at lower pulse intensities even though the parameter $\chi$ would reach $\sim 10$ without RD.

The ponderomotive side scattering is an important effect that cannot be easily incorporated within the quantum approach. Moreover, the Volkov solution cannot in principle describe this process, since an electron has no trajectory in this approach. However, the ponderomotive scattering is an important process in the laser-particle interaction. Figure 5 illustrates the trajectory of an electron scattered by an intense laser pulse. The calculation is performed for $w_{0}=5 \mu \mathrm{m}$; however, even a small shift of the electron $2 \mu \mathrm{m}$ in the transverse direction certainly 


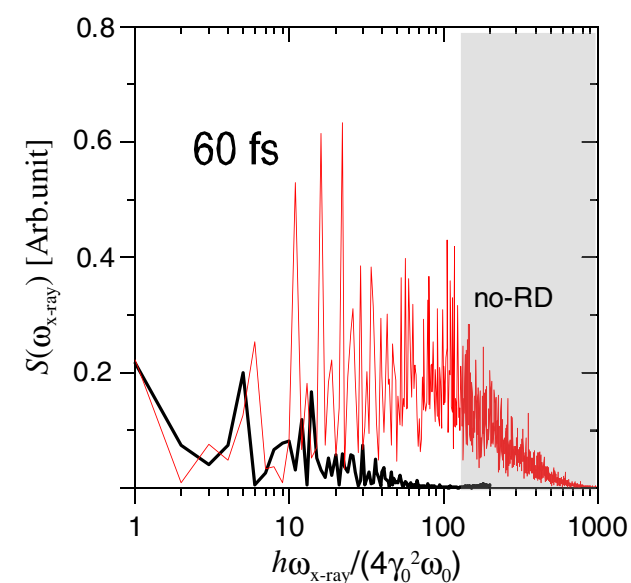

(a)

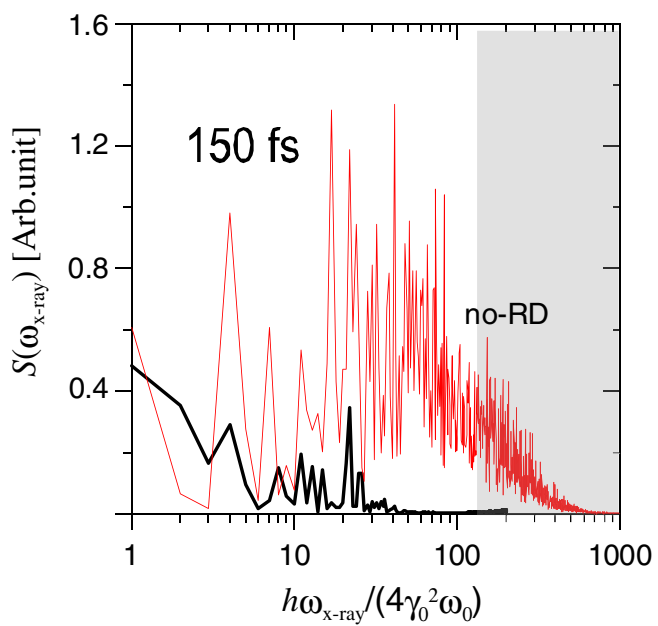

(c)

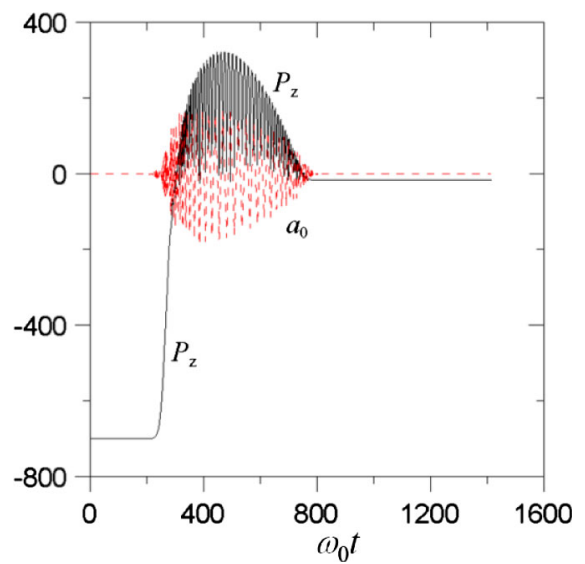

(b)

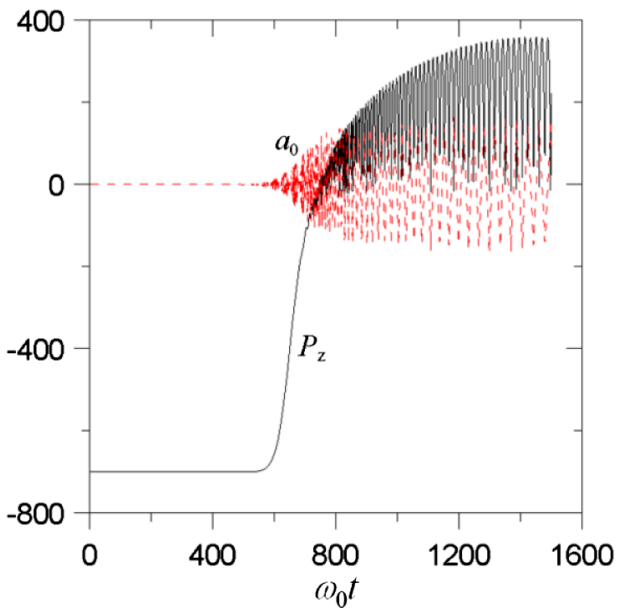

(d)

FIG. 2. Spectra of backward scattering $(\theta=\pi)$ with $a_{0}=200$ Gaussian pulses of duration 60 fs (a) and 150 fs (c) for a head-on collision, $\gamma=700$, with (red line) and without (black line) radiation damping. (b), (d) show the pulse field, $a_{0}(t)$ (red line), seen by the electron during the interaction and the longitudinal momentum (black line), $P_{\mathrm{z}^{\prime}}$, of that electron.

results in a change of its trajectory and resulting spectrum: the total spectral power decreases by more than a factor of 2 . The most dense electron beams are generated by laser wake field acceleration [15]. However, even these beams have a typical diameter $\sim 10 \mu \mathrm{m}$. This means the calculation of the scattered spectrum without the side scattering of the electron during the interaction will result in an incorrect spectrum and total radiated power. We anticipate that the classical calculations may provide reasonable spectral data even for very high energy electron beams. In Fig. 6 the backward spectrum is given for an electron with an initial energy of $\varepsilon \sim 8 \mathrm{GeV}$. One can see the "quantum" area indicated by the grey color contains a relatively low amount of energy and that the classical approximation is suitable for electron motion calculations.

The results of the calculation show that efficient Compton scattering in high fields is impossible due to the classical radiation damping and/or side scattering by the ponderomotive force. A partial solution of the problem lies in the use of two colliding laser pulses. In conventional accelerators the radiation losses are compensated by extra acceleration of electron beams [3]. In vacuum, an electron can be accelerated shortly by a copropagating laser pulse [16] (see also [17]). This acceleration undulates having a period, which increases with $\gamma$ and $a_{0}$. A proper choice is to make the copropagating laser pulse as the electron booster and compensate the energy losses for the radiation during the scattering of the counter-propagating laser pulse. The maximum momentum of an electron driven by a copropagating plane wave with $a_{0 \mathrm{D}}$ could exceed the initial momentum,

$$
\begin{aligned}
p_{Z}= & 0.5 m c\left\{1-\left(\gamma_{0}-\sqrt{\gamma_{0}^{2}-1}\right)\right. \\
& \left.+a_{o D}^{2} \sin ^{2}[\omega(t-z / c)]\right\} /\left(\gamma_{0}-\sqrt{\gamma_{0}^{2}-1}\right),
\end{aligned}
$$




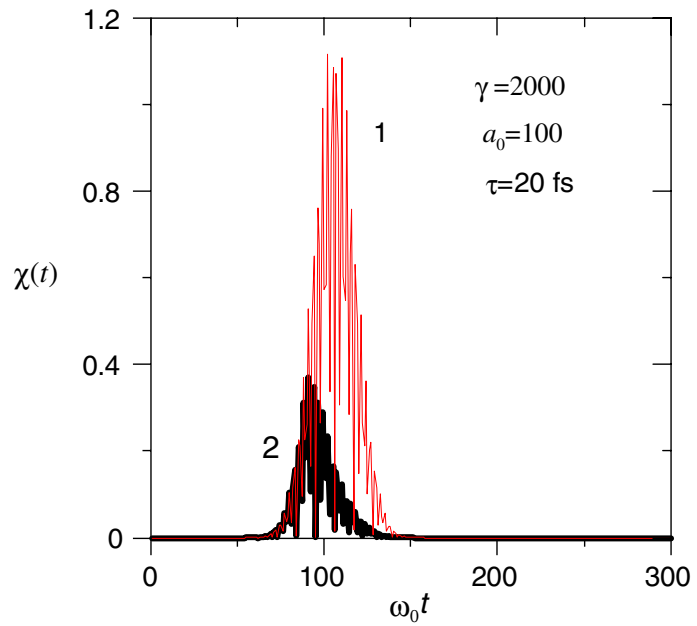

FIG. 3. Time evolution of the $\chi$ parameter for an electron $\gamma=2000$ without (1) (red curve) and with (2) (black curve) radiation damping in a 20 fs Gaussian pulse with $a_{0}=100$; head-on collision.

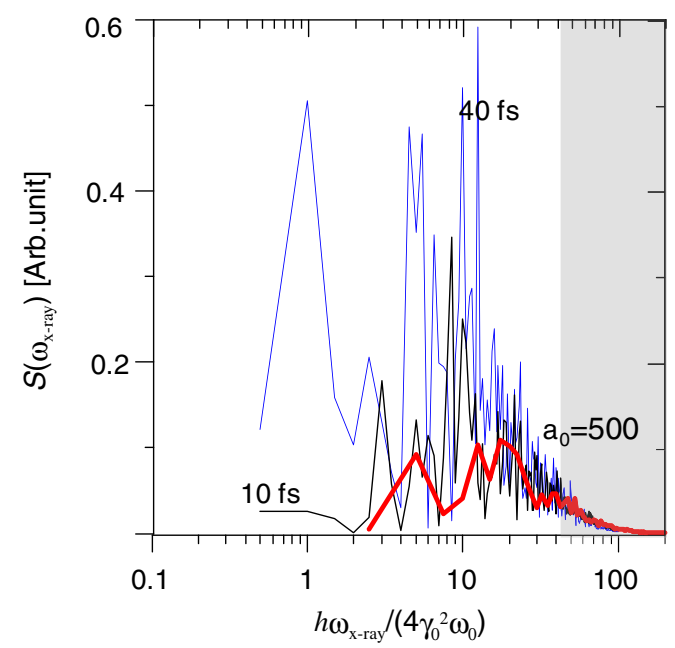

FIG. 4. Spectra of backward scattering $(\theta=\pi)$ with $a_{0}=100$ Gaussian pulses of duration 10 fs (black curve) and 40 fs (blue curve) for a head-on collision, $\gamma=2000$, with radiation damping, and (red curve) 10 fs laser pulse with $a_{0}=500$.

and, therefore, enhance the Compton scattering. The maximum value $p_{Z \max } \approx m c \gamma_{0} a_{0 D}^{2}$ can be achieved when $T_{\max }(1-\langle v\rangle / c) \approx \pi / 2$ where $\langle v\rangle$ is the average velocity of the electron [7]. In a focused laser pulse, the maximum electron momentum, being restricted by the pulse Rayleigh length, $L_{\mathrm{R}}$ (which is typically less than $c T_{\max }$ ), still exceeds the initial momentum [16]. However, the aim of a booster is not to increase the electron energy but to compensate the friction force. The maximum driving force of a pulse is $\left|f_{D}\right|=|\vec{v} \times \vec{B} / c| \approx B$. Therefore, the radiation friction can be overcome if the ratio of the forces $\kappa \approx 1.2 \times$ $10^{-8}\left(\gamma_{0} a_{0 C}\right)^{2} / a_{0 D}$ is less than unity (here $a_{0 \mathrm{C}}$ is the intensity of the scattered laser pulse). Because of a small numerical

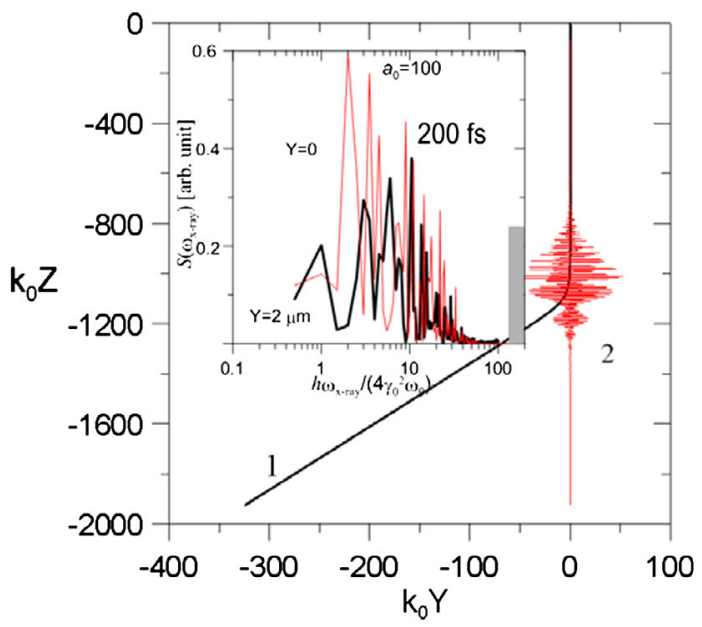

FIG. 5. Trajectory and backward spectra (insert) for $a_{0}=100$ and $\tau=200$ fs for a collision with a $2 \mu \mathrm{m}$ transverse shift (black curves) and for a head-on collision (red curves). Since the trajectory for the head-on collision is a straight line we give instead the pulse field evolution for this case (red curve).

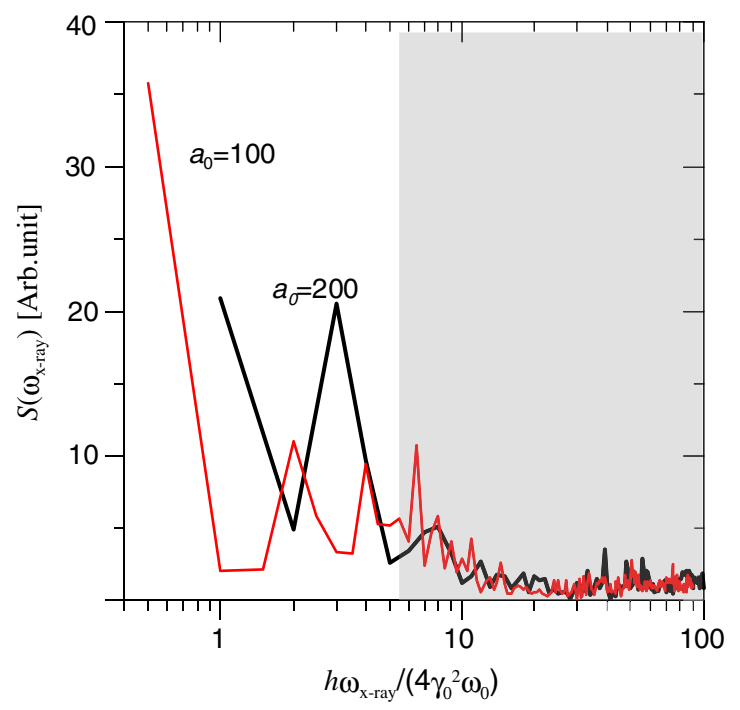

FIG. 6. Spectra of backward scattering $(\theta=\pi)$ with $a_{0}=100$ (red curve) and $a_{0}=200$ (black curve) Gaussian pulses of duration $20 \mathrm{fs}$ for a head-on collision with $\gamma=16000$ ( $\varepsilon \sim 8 \mathrm{GeV}$ ) with radiation damping. The number of spectral points is the same in both cases, $M=2000$, while the maximum frequency is different resulting in poorer resolution for $a_{0}=200$ at lower frequencies.

factor, the parameter is small for a wide range of electron energies and intensities of scattered laser pulses. In Fig. 7, a sample of the evolution of the electron longitudinal momentum in the two pulse scheme is presented. Both pulses have the same intensity $a_{0}=100$ and duration $\tau=10 \mathrm{fs}$, and both are focused to $w_{0}=5 \mu \mathrm{m}$. One can see that the radiation damping is compensated by the copropagating pulse acceleration and that Compton scattering in the vicinity 


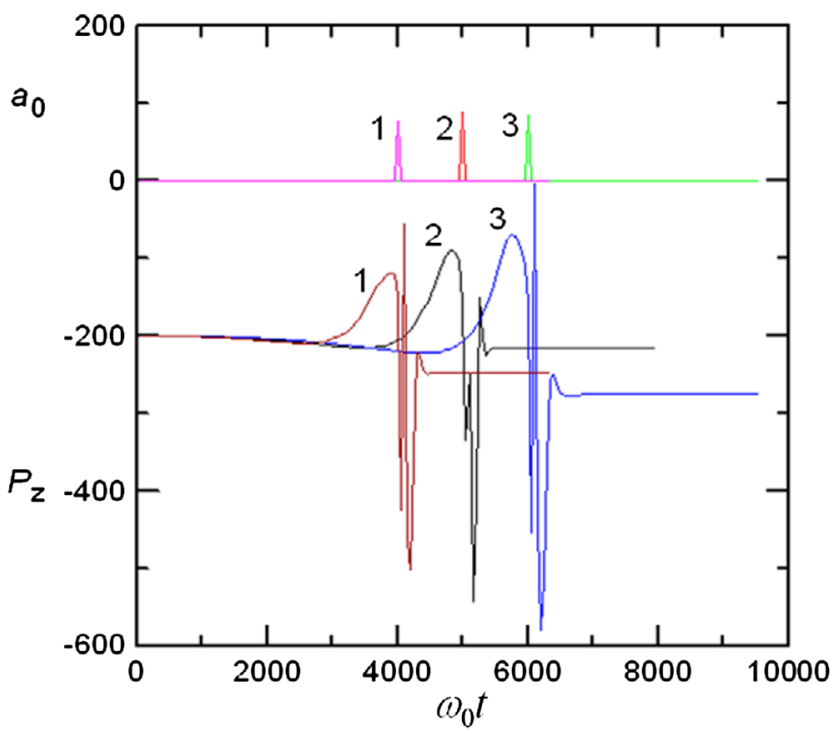

FIG. 7. Evolution of the electron longitudinal momentum in the two pulse scheme: both pulses have $a_{0}=100, \tau=10 \mathrm{fs}$, and are focused to $w_{0}=5 \mu \mathrm{m}$, head-on collision; (1), (2), (3) are given for different delays between the pulses: 0,45 , and $90 \mathrm{fs}$.

of the maximum laser intensity occurs at high electron energy even exceeding the initial energy. In Fig. 8 the spectra of backward scattering with respect to the laser propagation direction $(\theta=\pi)$ are presented for this case. However, if the scattered laser pulse is focused to $w_{0}=5 \mu \mathrm{m}$, the driving pulse is a plane wave with $a_{0 \mathrm{D}}=100$ and duration $10 \mathrm{fs}$. The plane wave is chosen to allow a comparison for backscattering: a focused driving laser pulse changes the trajectory

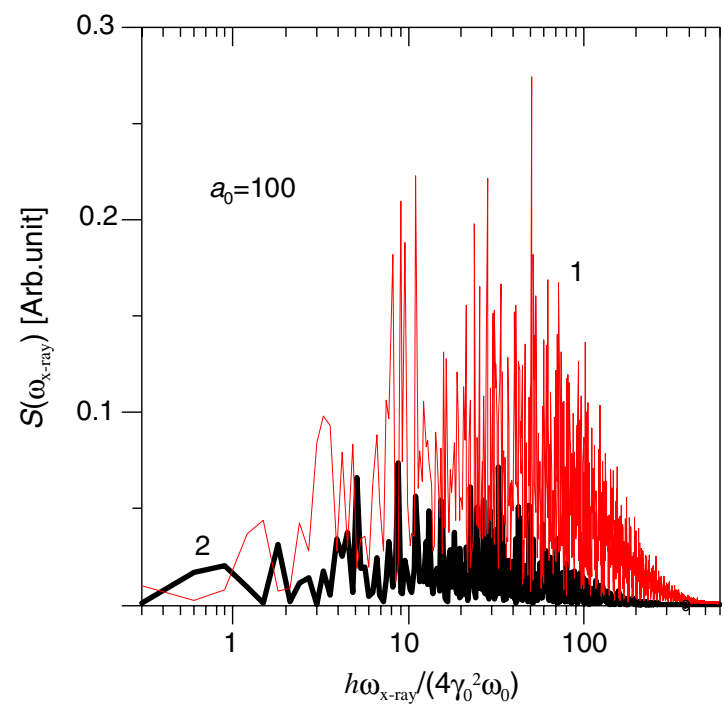

FIG. 8. Spectra of backward scattering $(\theta=\pi)$ in the two pulse scheme (1) (red curve) for the time delay $45 \mathrm{fs}$ as in Fig. 7 (2) (both pulses have $a_{0}=100, \tau=10 \mathrm{fs}$, the scattered pulse is focused to $w_{0}=5 \mu \mathrm{m}$, the driving pulse is a plane wave) and with only the scattered pulse (2) (black curve). of a boosted electron and, therefore, changes the angle of Compton scattering.

One can see that the spectrum of a $100 \mathrm{MeV}$ electron with the booster is similar to that without the radiation damping. However for $10 \mathrm{GeV}$ level electrons, efficient boosting requires a very large intensity for the driving pulses, of the order of $I=10^{26} \mathrm{~W} / \mathrm{cm}^{2}$, which is not achievable nowadays. The realization of this method for $\mathrm{GeV}$ electrons is difficult from not only a practical standpoint; the applicability of the classical approach for this case requires special analysis including the quantum approach. However, the conventional quantum approach based on the Volkov's solution is not correct for this case. The proper theory of the strong radiation regime for two pulses has yet to be developed. Nevertheless, since the quantum effects reduce the particle radiation, a laser pulse can be a particle booster in the strong, and even the quantum, radiation regime with the cascade scattering. This will allow the interaction of relativistic electrons with the main body of high intensity, $a_{0}>50$, laser pulses. The scheme can be organized similarly to that proposed in Ref. [18]. We consider here only head-on collisions. At finite collision angle the booster method is still efficient. However, getting to the quantum regime in this case becomes more problematic.

\section{CONCLUSION}

In conclusion, we have analyzed the cascade scattering of intense laser light by a relativistic electron in the regime of strong radiation damping in the framework of the classical approach. We have restricted our calculations to the regime where the radiation is strong, but the emission is located in the classical part of radiation spectrum, $\left\langle h \Omega_{\text {x ray }}\right\rangle<m c^{2}(\gamma-1)$. Our results have shown that, nowadays, the classical method still allows a self-consistent analysis of the interaction of relativistic particles with an intense laser field including the ponderomotive side scattering and electron vacuum acceleration. It has been shown that the classical approach remains valid at much higher laser intensities and particle energies due to the effect of the radiation damping resulting in much lower frequencies of emitted $\mathrm{x}$ rays. The parameter $\chi$ remains much less than unity due to the radiation damping even for $\mathrm{GeV}$ level electrons with laser intensities $\sim 10^{23}-10^{24} \mathrm{~W} / \mathrm{cm}^{2}$. In the case of plasma produced by intense short laser pulses, the quantum condition is even softer, because plasma electrons acquire their energy from the laser pulses starting from the classical regime.

It has been demonstrated that an experimental realization of the scattering by relativistic electrons at $a_{0 \mathrm{C}} \geq 100$ is impossible due to the radiation damping and ponderomotive sidescattering. Even high energy electrons lose their energy far before the maximum of the laser field is reached. The "two pulse scheme" with a copropagating laser pulse as an electron booster is proposed to solve the problem of 
nonlinear Compton scattering at high $a_{0 \mathrm{C}}$. The ponderomotive acceleration of electrons can overcome the radiation damping of electrons with energy lower than $1 \mathrm{GeV}$ with a practical intensity of the boosting laser pulse and an intensity of the scattered laser pulse exceeding $a_{0 \mathrm{C}}=100$. At higher energies the necessary intensities increase as $a_{0 \mathrm{D}} \sim \gamma_{0}^{2}$. Moreover for this case, a comprehensive quantum analysis requires more sophisticated approaches that have yet to be developed.

[1] G. Mourou, T. Tajima, and S. V. Bulanov, Rev. Mod. Phys. 78, 309 (2006); M. Marklund and P. Shukla, ibid. 78, 591 (2006); Y. I. Salamin, S. X. Hu, K. Z. Hatsagortsyan, and C. H. Keitel, Phys. Rep. 427, 41 (2006); A. Di Piazza, C. Muller, K. Z. Hatsagortsyan, and C. H. Keitel, Rev. Mod. Phys. 84, 1177 (2012).

[2] J. Colgan, J. Abdallah, A. Y. Faenov et al., Phys. Rev. Lett. 110, 125001 (2013); A. Zhidkov, J. Koga, A. Sasaki, and M. Uesaka, Phys. Rev. Lett. 88, 185002 (2002); S. V. Bulanov, T.Zh. Esirkepov, J. K. Koga, and T. Tajima, Plasma Phys. Rep. 30, 196 (2004); T. Nakamura, J. K. Koga, T.Zh. Esirkepov, M. Kando, G. Korn, and S. V. Bulanov, Phys. Rev. Lett. 108, 195001 (2012); C. P. Ridgers, C. S. Brady, R. Duclous, J. G. Kirk, K. Bennett, T. D. Arber, A. P. L. Robinson, and A. R. Bell, Phys. Rev. Lett. 108, 165006 (2012); C. S. Brady, C. P. Ridgers, T. D. Arber, A. R. Bell, and J. G. Kirk, ibid. 109, 245006 (2012); F. V. Hartemann, Report No. LLNL-PROC-409586, 2008.

[3] I. M. Ternov, Usp. Fiz. Nauk 165, 429 (1995).

[4] V. B. Berestetskii, E. M. Lifshitz, and L. P. Pitaevskii, Quantum Electrodynamics (Butterworth-Neinemann, Jordan Hill, Oxford, 1982), Vol. 4.

[5] A. I. Nikishov and V. I. Ritus, Sov. Phys. JETP 19, 529 (1964); 19, 1191 (1964); I. Goldman, Sov. Phys. JETP 19, 954 (1964).

[6] N. B. Narozhny, S. S. Bulanov, V. D. Mur, and V. S. Popov, Phys. Lett. A 330, 1 (2004); S. S. Bulanov, V. D. Mur, N. B. Narozhny, and V.S. Popov, JETP Lett. 80, 734 (2004); JETP 129, 14 (2006); S. S. Bulanov, A. M. Fedotov, and F. Pegoraro, JETP Lett. 80, 734 (2004); Phys. Rev. E 71, 016404 (2005); I. V. Sokolov, J. A. Nees,
V. P. Yanovsky, N. M. Naumova, and G. A. Mourou, Phys. Rev. E 81, 036412 (2010); I. V. Sokolov, N. M. Naumova, J. A. Nees, and G. A. Mourou, Phys. Rev. Lett. 105, 195005 (2010); S. S. Bulanov, C. B. Schroeder, E. Esarey, and W. P. Leemans, Phys. Rev. A 87, 062110 (2013).

[7] L. Landau and E. M. Lifshitz, The Classical Theory of Fields (Pergamon Press, Oxford, 1975), p. 227.

[8] E. Esarey, S. K. Ride, and P. Sprangle, Phys. Rev. E 48, 3003 (1993).

[9] P. Panek, J. Z. Kaminski, and F. Ehlotzky, Laser Phys. 13, 457 (2003).

[10] S. V. Bulanov, T. Zh. Esirkepov, M. Kando, J. K. Koga, and S. S. Bulanov, Phys. Rev. E 84, 055605 (2011); A. R. Bell and J. G. Kirk, Phys. Rev. Lett. 101, 200403 (2008); J. G. Kirk, A. R. Bell, and I. Arka, Plasma Phys. Controlled Fusion 51, 085008 (2009).

[11] F. V. Hartemann and A. K. Kerman, Phys. Rev. Lett. 76, 624 (1996); G. A. Krafft, Phys. Rev. Lett. 92, 204802 (2004); A. G. R. Thomas, C. P. Ridgers, S. S. Bulanov, B. J. Griffin, and S.P. D. Mangles, Phys. Rev. X 2, 041004 (2012); C. Harvey, T. Heinzl, A. Ilderton, and M. Marklund, Phys. Rev. Lett. 109, 100402 (2012); F. Mackenroth and A. Di Piazza, Phys. Rev. A 83, 032106 (2011).

[12] W. H. Press, S. A. Teukilsky, W. T. Vettering, and B. P. Flannery, Numerical Recipes in Fortran (University Press, Cambridge, 1992), 2nd ed., p. 704.

[13] A. Yariv, Optical Electronics (Holt, Rinehart and Winston, New York, 1985).

[14] I. Ghebregziabher, B. A. Shadwick, and D. Umstadter, Phys. Rev. ST Accel. Beams 16, 030705 (2013); F. V. Hartemann, F. Albert, C. W. Siders, and C. P. Barty, Phys. Rev. Lett. 105, 130801 (2010).

[15] T. Tajima and J. M. Dowson, Phys. Rev. Lett. 43, 267 (1979).

[16] G. V. Stupakov and M. S. Zolotorev, Phys. Rev. Lett. 86, 5274 (2001); P. X. Wang, Y. K. Ho, X. Q. Yuan, Q. Kong, N. Cao, A. M. Sessler, E. Esarey, and Y. Nishida, Appl. Phys. Lett. 78, 2253 (2001).

[17] A. D. Debus, M. Bussmann, M. Siebold, A. Jochmann, U. Schramm, T. E. Cowan, and R. Sauerbrey, Appl. Phys. B 100, 61 (2010).

[18] J. Faure, C. Rechatin, A. Norlin, A. Lifschitz, Y. Glinec, and V. Malka, Nature (London) 444, 737 (2006). 